\title{
High-Sensitivity Compton Imaging with Position-Sensitive Si and Ge Detectors
}

K. Vetter, M. Burks, C. Cork, M. Cunningham, D. Chivers, E. Hull, T. Krings, H. Manini, L. Mihailescu, K. Nelson, D. Provtic, J. Valentine, D. Wright

June 19, 2006

Nuclear Instruments and Methods in Physics Research, Section A 
This document was prepared as an account of work sponsored by an agency of the United States government. Neither the United States government nor Lawrence Livermore National Security, LLC, nor any of their employees makes any warranty, expressed or implied, or assumes any legal liability or responsibility for the accuracy, completeness, or usefulness of any information, apparatus, product, or process disclosed, or represents that its use would not infringe privately owned rights. Reference herein to any specific commercial product, process, or service by trade name, trademark, manufacturer, or otherwise does not necessarily constitute or imply its endorsement, recommendation, or favoring by the United States government or Lawrence Livermore National Security, LLC. The views and opinions of authors expressed herein do not necessarily state or reflect those of the United States government or Lawrence Livermore National Security, LLC, and shall not be used for advertising or product endorsement purposes. 


\title{
High-Sensitivity Compton Imaging with Position- Sensitive Si and Ge Detectors
}

\author{
K. Vetter ${ }^{1}$, M. Burks ${ }^{1}$, C. Cork ${ }^{1}$, M. Cunningham ${ }^{1}$, D. Chivers ${ }^{1}$, E. Hull ${ }^{1}$, T. Krings ${ }^{2}$, H. \\ Manini $^{1}$, L. Mihailescu ${ }^{1}$, K. Nelson ${ }^{1}$, D. Protic ${ }^{2}$, J. Valentine ${ }^{1}$, and D. Wright ${ }^{1}$ \\ ${ }^{1}$ Lawrence Livermore National Laboratory, Livermore CA 94550, USA \\ ${ }^{2}$ Institut für Kernphysik, Forschungszentrum Jülich, 52425 Jülich, Germany
}

\begin{abstract}
We report on the development of high-sensitivity and compact Compton imaging systems built of large and position-sensitive $\mathrm{Si}(\mathrm{Li})$ and HPGe detectors. The primary goal of this effort is to provide improved capabilities in the passive detection of nuclear materials for homeland security. Our detectors are implemented in double-sided strip configuration which - along with digital signal processing - provides energies and three-dimensional position information of individual gamma-ray interactions. Gamma-ray tracking algorithms are then determining the scattering sequence of the gamma ray which in turn allows us - employing the Compton scattering formula - to reconstruct a cone of possible incident angles and ultimately an image. This Compton imaging concept enables large-field-of-view gamma-ray imaging without the use of a heavy collimator or aperture. The intrinsically high energy resolution of the detectors used, the excellent position resolution we have demonstrated, both combined with the high efficiency of large-volume detectors is the basis for high Compton imaging sensitivity. These capabilities are being developed to identify and localize potential threat sources and to potentially increase the sensitivity in detecting weak sources out of the midst of natural, medical, or commercial sources. Gamma-ray imaging provides a new degree of freedom to distinguish between spatial and temporal background fluctuations and compact threat sources.
\end{abstract}

\section{Introduction}

Gamma-ray imaging is an established tool in biomedical applications, nuclear medicine, and astrophysics. More recently however, the impact of gammaray imaging for homeland security applications has been recognized as well $[1,2]$. Here, the goal is to provide improved capabilities to detect, localize, and characterize nuclear materials by passive means without compromising the environment. One of the outstanding challenges in homeland security is the detection and identification of potential nuclear and radiological threats in the midst of a sea of non-threat objects, which consists of legitimate radioactive objects commonly found in commerce and environment. Current passive radiation detection technologies lack in sensitivity and specificity in uniquely detecting, identifying, and localizing radioactive sources of interest. This deficiency is multiplied in wide-area surveillance applications in which weak

threat sources have to be distinguished from spatial and temporal background fluctuations and in the midst of legitimate radioactive sources.

We are developing highly sensitive Compton imaging system to address these challenges by providing a potentially compact instrument with excellent energy resolution, good imaging performance and large field-ofview. More specifically, we are developing Compton imaging systems which are built of large-volume and high-resolution $\mathrm{Si}(\mathrm{Li})$ and $\mathrm{HPGe}$ detectors. These detectors have intrinsically good energy resolution and are built in double-sided strip configurations, which - along with digital signal processing - provides excellent three-dimensional position resolution. These fundamental characteristics allow us to measure individual gamma-ray interactions and to 
time order them through gamma-ray tracking algorithms. These capabilities in addition to using large-volume and therefore efficient detectors provide the basis for high sensitivity Compton imaging.

The concept of Compton imaging was proposed many years ago $[3,4]$, however, only recent advances in detector manufacture and compact electronics allows us now to realize instruments with competitive sensitivity. Below, we will first briefly describe our approach for Compton imaging and our instrument. We will then present results of angular resolution measurements we obtained for a range of energies and source locations as well as preliminary results to image extended sources. We have invested significant effort in advanced data analysis ranging from simple pulse-shape analysis to advanced image reconstruction as well modeling of a large variety of Compton imaging implementations and scenarios which we can't discuss here giving the scope of this paper.

\section{The Si-Ge Compton imaging instrument}

We have performed extensive MonteCarlo simulations to determine a path to demonstrate the potential capabilities of Compton imaging for gamma-ray energies ranging from about $150 \mathrm{keV}$ to about $3 \mathrm{MeV}$, the range most interesting for homeland security. Taking into account fundamental physics and technological limitations, such as the intrinsic momentum of Compton scattered electrons, or achievable position and energy resolution of specific detector materials and implementations, we determined that a semi-conductor detector system built of low- $Z$ and high- $Z$ materials is currently the most promising approach. Following these calculations, we are developing a hybrid Compton imager consisting of low-Z Si detectors and higher-Z Ge detectors. Figure 3 shows results of Monte-Carlo simulations to estimate the efficiency for different thicknesses of each layer and gamma-ray energies. The separation between each layer was $3 \mathrm{~cm}$. From this figure we can see that a $\mathrm{Si}$ thickness of at least $20 \mathrm{~mm}$ and a Ge thickness of $30 \mathrm{~mm}$ provide close to maximum efficiency. For higher energies the efficiency can be increased with larger thickness, however, gamma rays interacting only in Ge can be used for efficient Compton imaging as well. In addition, the drop in efficiency for higher gamma-ray energies is small compared with the drop in collimatorbased imagers.

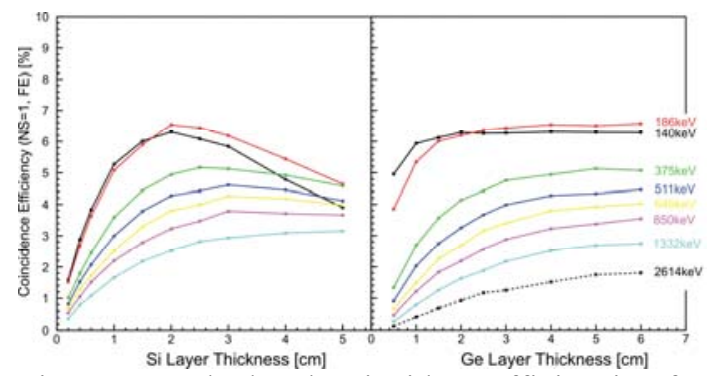

Figure 1: Calculated coincident efficiencies for hybrid Compton imager as a function of $\mathrm{Si}$ and Ge detector thickness and incident gamma-ray energy. Only gamma rays which interact exactly once in the Si layer and deposit the full energy in the instrument are considered.

As a result of these calculations we are developing a hybrid Compton imaging system which consists of two layers of $10 \mathrm{~mm}$ thick $\mathrm{Si}(\mathrm{Li})$ detectors in front of two $15 \mathrm{~mm}$ thick HPGe detectors. We are currently assembling such a system. As a first step however, we have built and demonstrated a hybrid system consisting of one $64 \times 64 \times 10 \mathrm{~mm}^{3} \mathrm{Si}(\mathrm{Li})$ and one $76 \times 76 \times 11 \mathrm{~mm}^{3} \mathrm{HPGe}$ detector. The detectors mounted in individual cryostats are shown in Figure 2. The complete system including data acquisition electronics and processing and monitoring computer is mounted on a cart to provide the ability to perform 
measurements easily in different locations.

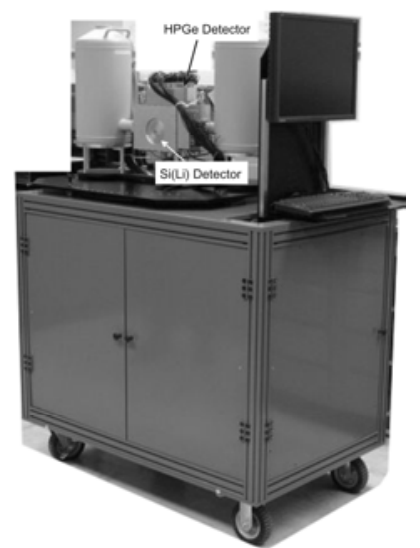

Figure 2: First Compact Compton Imager (CCI1). The complete system including data acquisition mounted in the cart.

All the results we will present in the following were obtained with this first Compact Compton Imager (CCI-1). Each detector is implemented in doublesided strip configuration with a pitch size of $2 \mathrm{~mm}$. The $\mathrm{Si}(\mathrm{Li})$ detector has 32 strips on each size with a gap between the strips of less then $50 \mu \mathrm{m}$, the HPGe has 39 strips on each side with a gap of $250 \mu \mathrm{m}$. The front faces of both detectors are separated by $60 \mathrm{~mm}$. Each strip is readout individually with compact but discrete preamplifiers. The signals are then digitized in a system of waveform digitizers manufactured by Struck Innovative Systems. The signals are sampled at $100 \mathrm{MHz}$ with a vertical resolution of 14 bit. With this system, we obtain energy resolution values in the order of $1.4 \mathrm{keV}$ and $1.7 \mathrm{keV}$ at $60 \mathrm{keV}$ and $1.9 \mathrm{keV}$ and $2.1 \mathrm{keV}$ at $662 \mathrm{keV}$ for the HPGe detector and the $\mathrm{Si}(\mathrm{Li})$ detector, respectively. Threedimensional positions are deduced by digital signal processing using net charge signals as well adjacent strips which display only transient charge signals. In this way we have demonstrated better than $0.5 \mathrm{~mm}$ resolution in each dimension. With more sophisticated processing algorithms we are able to obtain these values throughout the detector volume, even for interactions close to the gap. To maximize the sensitivity we are currently developing tools that allow us to disentangle multiple interactions in close vicinity, even in one strip. We have developed accurate models to describe the charge transport in our detectors and are now able to calculate signal shapes with very good agreement with measured signals. This effort will ultimately allow us to fit measured signals with calculated signals to decompose multiple into single interactions. Independently, we have developed data processing schemes and gamma-ray tracking schemes, which allow us to reconstruct between $60 \%$ and $80 \%$ of all coincident and full-energy events.

\section{Angular resolution and image reconstruction}

With CCI-1, we have performed a range of image measurements employing point and extended ${ }^{22} \mathrm{Na},{ }^{137} \mathrm{Cs}$ and ${ }^{152} \mathrm{Eu}$ sources at different incident angles. To provide a flavor of these measurements we present two aspects, the angular resolution and image reconstruction of a ${ }^{152} \mathrm{Eu}$ point and line source. As a

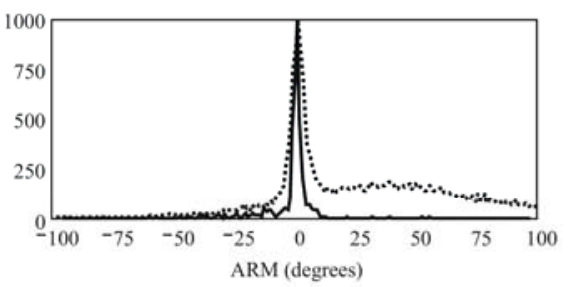

Figure 3: Experimental ARM distributions for $244 \mathrm{keV}$ (dashed line) and $1408 \mathrm{keV}$ (solid line).

measure of the angular resolution we use the commonly used Angular Resolution Measure (ARM) as shown in Figure 3 for $244 \mathrm{keV}$ and $1408 \mathrm{keV}$. We use as measure the FWHM of the background 

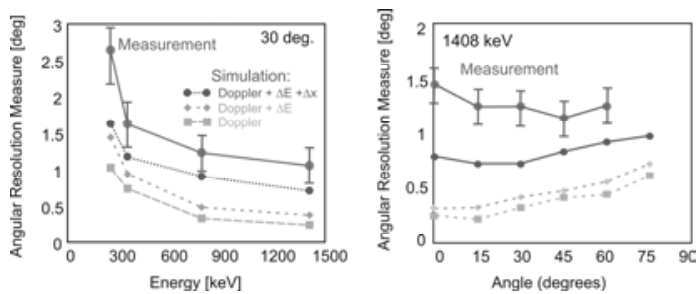

Figure 4: Measured ARM values for different energies (left) and source locations (right) in comparison with calculations.

subtracted ARM distributions, which are shown in Figure 4. For comparison, calculated ARM values are shown assuming uncertainties due to the intrinsic electron momentum (Doppler) only, with the measured energy resolution, and finally also with the position uncertainty. We achieve very good angular resolution values which are dominated by the intrinsic Doppler broadening. A possible explanation for the small deviation between measured and calculated ARM could be a misalignment of the $\mathrm{Si}$ and Ge detector of the order of $0.5 \mathrm{~mm}$.

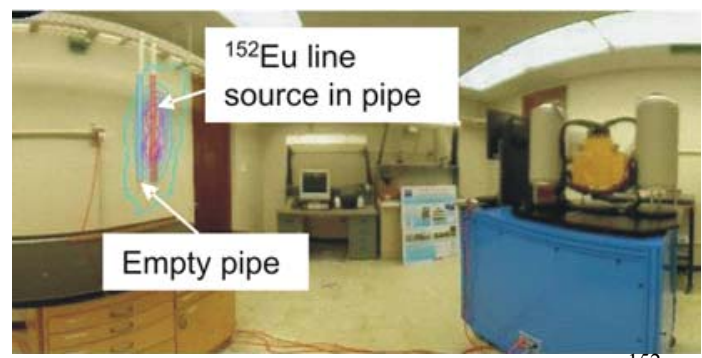

Figure 5: Gamma-ray image of a hidden ${ }^{152} \mathrm{Eu}$ line source merged with a visual image. Visually, it is not possible to determine which pipe contains the radioactive material. With our gamma-ray imager not only can the pipe be identified but also the source extension can be determined as well as the radioisotope.

Figure 5 shows one example of the extended source measurements with a ${ }^{152} \mathrm{Eu}$ line source is hidden in one of several pipes.

\section{Other applications}

Our approach of gamma-ray tracking based Compton imaging can be applied not only to national security applications such as homeland security or in biomedical imaging but the underlying concept will help to improve the sensitivity to detect gamma rays for basic nuclear physics experiment or will help in the search for the neutrino-less double beta decay, e.g. in segmented ${ }^{76} \mathrm{Ge}$ detectors, to ultimately measure the mass of neutrinos.

\section{Conclusions and outlook}

We have demonstrated excellent performance of a first-generation, hybrid Compton imaging system built of one large DSSD $\mathrm{Si}(\mathrm{Li})$ and one large DSSD HPGe detector. We are now building a second generation instrument consisting of two large DSSD $\mathrm{Si}(\mathrm{Li})$ and two larger DSSD HPGe detectors in closer geometry than before to significantly increase the efficiency and therefore the overall sensitivity of such an instrument. This system will allow us to perform real-time imaging of radioactive sources at distances larger than $10 \mathrm{~m}$. Ultimately, we envision a compact Compton imaging module of these four detectors which can be scaled up to larger dimensions to extend the range beyond $10 \mathrm{~m}$.

\section{Acknowledgments}

This work was performed under the auspices of the U.S. Department of Energy by University of California Lawrence Livermore National Laboratory under contract No. W-7405Eng-48

\section{References}

[1] K. P. Ziock et al., IEEE Trans. Nucl. Sci., $51-5$ (2004) 2238

[2] T. Niedermayr et al., Nucl. Instr. Meth. 553-3 (2005) 501

[3] R.W. Todd, J.M. Nightingale, and D.B. Everett, Nature 251 (1974) 132

[4] V. Schoenfelder et al., Nucl. Instr. Meth. 107 (1973) 385 